\title{
Novel and Bioactive Compounds from the Malayan Flora; Recent Findings and Challenges
}

\author{
Khalijah Awang*
}

Chemistry Department, Faculty of Science, University Malaya, 50603 Kuala Lumpur; E-mail: khalijah@um.edu.my

Malaysia is one of the seventeen megadiverse countries in the world. Its flora is home to an estimated 15,000 higher plant species and it is widely known that plants are efficient manufacturers of compounds with highly diversed molecular structures and an unending array of biological activities.

Therefore, it is logical to assume that a multitude of molecules and their activities are yet to be discovered from this beautiful and immense flora. In fact, our experience with plants from various families; Annonaceae, Apocynaceae, Lauraceae, Meliaceae and Rubiaceae, have evidenced that many types of interesting and unique compounds were synthesized by these plants; alkaloids, triterpenes, limonoids, lignans, coumarins and many others.

In this presentation, recent findings on some novel and bioactive compounds from some higher plants shall be communicated which includes some mechanisms in their mode of action for example the novel malayanine A and B from Chisocheton erythrocarpus and the neuroprotective and acetylcholinesterase inhibiting mesuagenins isolated from Mesua species.

In addition, as Natural Products and Drug Discovery is an important research area in Malaysia, the challenges and possible directions in this field shall be discussed briefly. For instance, how can we, the natural products scientists relate our findings and knowledge to the society and then further contribute to the enhancement of its well-being?

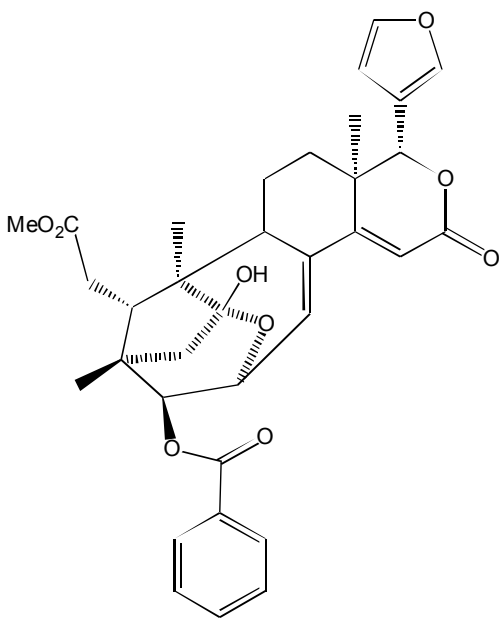

Malayanine A

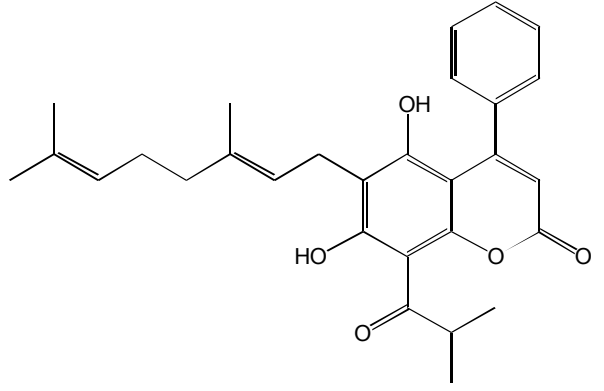

Mesuagenin $\mathrm{C}$ 